\title{
Regular fuzzy equivalences on multi-mode multi-relational fuzzy networks
}

\author{
Miroslav Ćirić, Jelena Ignjatović, Ivan Stanković \\ University of Niš, Faculty of Sciences and Mathematics, Višegradska 33, 18000 Niš, Serbia
}

\begin{abstract}
In this paper we introduce the concepts of a multimode multi-relational fuzzy network and a regular fuzzy equivalence on such a network, and provide procedures for computing the greatest regular fuzzy and crisp equivalences contained in a given tuple of fuzzy equivalences.
\end{abstract}

Keywords: Multi-mode fuzzy network, regular fuzzy equivalence, fuzzy relation equation, residual of fuzzy relations.

\section{Introduction}

Social network analysis has originated as a branch of sociology and mathematics which provides formal models and methods for the systematic study of social structures. Social networks share many common properties with other types of networks, and methods of social network analysis are nowadays applied to the analysis of networks in general, including many kinds of networks that arise in computer science, physics, biology, etc., such as the hyperlink structure on the Web, the electric grid, computer networks, information networks or various large-scale networks appearing in nature.

In large and complex networks it is impossible to understand the relationship between each pair of individuals, but to a certain extent, it may be possible to understand the system, by classifying individuals and describing relationships on the class level. For instance, individuals in the same class can be considered to occupy the same position, or play the same role in the network. The main aim of the positional analysis of networks is to find similarities between individuals which have to reflect their position in a network. These similarities have been formalized first by Lorrain and White [17] by the concept of a structural equivalence. Informally speaking, two individuals are considered to be structurally equivalent if they have identical neighborhoods. However, in many situations this concept has shown oneself to be too strong. Weakening it sufficiently to make it more appropriate for modeling social positions, White and Reitz [21] have introduced the concept of a regular equivalence, where two individuals are considered to be regularly equivalent if they are equally related to equivalent others. Regular equivalences also play an important role in the blockmodeling, a method of data reduction which reduces redundant elements of a network to yield a simplified model of relationships between types of elements (cf. [1, 7, 9]).

Regular equivalences have been studied mainly in the context of one-mode social networks, consisting of a single set of entities and ties between these entities of the same or different types. To a lesser extent, regular equivalences and blockmodeling have been studied in the context of two-mode networks, which consist of two sets of entities and ties from one to another set (cf. [1, 4, 7, 8, 9, 18, 19, 20]). However, in real situations we often encounter much more complex networks consisting of multiple sets of entities and ties inside and between some of them. The main aim of this paper is to provide a general mathematical model for the study of such complex networks, which are called multi-mode networks, to introduce the concept of a regular equivalence on these networks, and to provide a procedure for computing these regular equivalences. It should be noted that a similar kind of networks, called multilevel networks, has been recently studied in [22]. In addition, we consider an even more general case, also very common in real situations, where the ties between entities are fuzzy. Such fuzzy social networks have been studied in $[6,10,11,12,14,15,16]$.

The methodology used here for computing regular fuzzy equivalences on multi-mode fuzzy networks is based on methods for finding the greatest solutions of so-called weakly linear systems of fuzzy relation inequalities and equations, developed in $[14,15,16]$, where residual of fuzzy relations play a key role. It is worth noting that this methodology has been previously shown to be very efficient in solving some fundamental problems of the theory of fuzzy automata, such as the reduction of the number of states and the problems of equivalence, simulation and bisimulation.

After this introductory section, in Section 2 we define the basic concepts of the theory of fuzzy sets and fuzzy relations that are used in further work. Then in Section 3 we define a multi-mode multi-relational fuzzy network and the concept of a regular fuzzy equivalence on such a network, and present the main results of the paper which provide a procedure for computing the greatest regular fuzzy and crisp equivalences on such networks.

The results obtained in this paper generalize the related results concerning both one-mode and twomode fuzzy networks. 


\section{Preliminaries}

In the paper we use complete residuated lattices as structures of membership values. A residuated lattice is an algebra $\mathcal{L}=(L, \wedge, \vee, \otimes, \rightarrow, 0,1)$ such that

(L1) $(L, \wedge, \vee, 0,1)$ is a lattice with the least element 0 and the greatest element 1 ,

(L2) $(L, \otimes, 1)$ is a commutative monoid with the unit 1

(L3) $\otimes$ and $\rightarrow$ form an adjoint pair, i.e., they satisfy the adjunction property: for all $x, y, z \in L$,

$$
x \otimes y \leqslant z \Leftrightarrow x \leqslant y \rightarrow z .
$$

If, additionally, $(L, \wedge, \vee, 0,1)$ is a complete lattice, then $\mathcal{L}$ is called a complete residuated lattice.

The operations $\otimes$ (called multiplication) and $\rightarrow$ (called residuum) are intended for modeling the conjunction and implication of the corresponding logical calculus, and supremum $(\bigvee)$ and infimum $(\bigwedge)$ are intended for modeling the existential and general quantifier, respectively. An operation $\leftrightarrow$ given by

$$
x \leftrightarrow y=(x \rightarrow y) \wedge(y \rightarrow x),
$$

called biresiduum (or biimplication), is used for modeling the equivalence of truth values. It can be easily shown that with respect to $\leqslant, \otimes$ is isotonic in both arguments, $\rightarrow$ is isotonic in the second and antitonic in the first argument. For other properties of complete residuated lattices we refer to $[2,3]$.

The most studied and applied structures of membership values, defined on the real unit interval $[0,1]$ with $x \wedge y=\min (x, y)$ and $x \vee y=\max (x, y)$, are the Eukasiewicz structure $(x \otimes y=\max (x+y-1,0)$ and $x \rightarrow y=\min (1-x+y, 1))$, the Goguen (product) structure $(x \otimes y=x \cdot y, x \rightarrow y=1$ if $x \leqslant y$ and $=y / x$ otherwise) and the Gödel structure $(x \otimes y=$ $\min (x, y), x \rightarrow y=1$ if $x \leqslant y$ and $=y$ otherwise). Another important set of truth values is the set $\left\{a_{0}, a_{1}, \ldots, a_{n}\right\}, 0=a_{0}<\cdots<a_{n}=1$, with $a_{k} \otimes a_{l}=a_{\max (k+l-n, 0)}, a_{k} \rightarrow a_{l}=a_{\min (n-k+l, n)} . \mathrm{A}$ special case of the latter algebras is the two-element Boolean algebra of classical logic with the support $\{0,1\}$. The only adjoint pair on this Boolean algebra consists of the classical conjunction and implication operations. This structure of truth values is called the Boolean structure.

In the sequel $\mathcal{L}$ will be a complete residuated lattice. A fuzzy subset of a set $A$ over $\mathcal{L}$, or simply a fuzzy subset of $A$, is any mapping from $A$ into $L$. Ordinary crisp subsets of $A$ are considered as fuzzy subsets of $A$ taking membership values in the set $\{0,1\} \subseteq L$. Let $f$ and $g$ be two fuzzy subsets of $A$. The equality of $f$ and $g$ is defined as the usual equality of mappings, i.e., $f=g$ if and only if $f(x)=g(x)$, for every $x \in A$. The inclusion $f \leqslant g$ is also defined pointwise: $f \leqslant g$ if and only if $f(x) \leqslant g(x)$, for every $x \in A$. Endowed with this partial order the set $L^{A}$ of all fuzzy subsets of $A$ forms a complete lattice, in which the meet (intersection) $\bigwedge_{i \in I} f_{i}$ and the join (union) $\bigvee_{i \in I} f_{i}$ of an arbitrary family $\left\{f_{i}\right\}_{i \in I}$ of fuzzy subsets of $A$ are mappings from $A$ into $L$ defined by

$$
\left(\bigwedge_{i \in I} f_{i}\right)(x)=\bigwedge_{i \in I} f_{i}(x), \quad\left(\bigvee_{i \in I} f_{i}\right)(x)=\bigvee_{i \in I} f_{i}(x),
$$

for all $x \in A$.

A fuzzy relation between sets $A$ and $B$ (in this order) is any fuzzy subset of $A \times B$, and the equality, inclusion (ordering), joins and meets of fuzzy relations are defined as for fuzzy sets. The set of all fuzzy relations between $A$ and $B$ will be denoted by $L^{A \times B}$. In particular, a fuzzy relation on a set $A$ is any fuzzy subset of $A \times A$, and the set of all fuzzy relations on $A$ is denoted by $L^{A \times A}$. The reverse or inverse of a fuzzy relation $\alpha \in L^{A \times B}$ is a fuzzy relation $\alpha^{-1} \in L^{B \times A}$ defined by $\alpha^{-1}(b, a)=\alpha(a, b)$, for all $a \in A$ and $b \in B$. A crisp relation is a fuzzy relation taking values only in the set $\{0,1\}$, and if $\alpha$ is a crisp relation between $A$ and $B$, then the expressions " $\alpha(a, b)=1$ " and " $(a, b) \in \alpha$ " will have the same meaning. For a fuzzy relation $\alpha \in L^{A \times B}$, the crisp relation $\alpha^{c}$ given by

$$
\alpha^{c}(a, b)= \begin{cases}1 & \text { if } \alpha(a, b)=1 \\ 0 & \text { otherwise }\end{cases}
$$

is called the crisp part of $\alpha$ (it is also known as the 1 -cut of $\alpha$ ). According to the above mentioned convention, we also write

$$
\alpha^{c}=\{(a, b) \in A \times B \mid \alpha(a, c)=1\} .
$$

For non-empty sets $A, B$ and $C$, and fuzzy relations $\alpha \in L^{A \times B}$ and $\beta \in L^{B \times C}$, their composition is a fuzzy relation $\alpha \circ \beta \in L^{A \times C}$ defined by

$$
(\alpha \circ \beta)(a, c)=\bigvee_{b \in B} \alpha(a, b) \otimes \beta(b, c),
$$

for all $a \in A$ and $c \in C$. When the underlying sets are finite, fuzzy relations can be interpreted as matrices with entries in $L$ and the composition of fuzzy relations can be interpreted as a kind of matrix product. It is easy to verify that the composition of fuzzy relations is associative and distributive over unions (joins) of fuzzy relations.

Let $A, B$ and $C$ be non-empty sets, $\lambda \in L^{A \times B}$, $\mu \in L^{B \times C}$ and $\eta \in L^{A \times C}$. The right residual of $\eta$ by $\lambda$ is a fuzzy relation $\lambda \backslash \eta \in L^{B \times C}$ defined by

$$
(\lambda \backslash \eta)(b, c)=\bigwedge_{a \in A} \lambda(a, b) \rightarrow \eta(a, c),
$$

for all $(b, c) \in B \times C$, and the left residual of $\eta$ by $\mu$ is a fuzzy relation $\eta / \mu \in L^{A \times B}$ defined by

$$
(\eta / \mu)(a, b)=\bigwedge_{c \in C} \mu(b, c) \rightarrow \eta(a, c)
$$

for all $(a, b) \in A \times B$. It is not hard to verify that the following residuation property (in some sources 
called the adjunction property) holds for arbitrary $\lambda \in L^{A \times B}, \mu \in L^{B \times C}$ and $\eta \in L^{A \times C}$ :

$$
\lambda \circ \mu \leqslant \eta \quad \Leftrightarrow \quad \mu \leqslant \lambda \backslash \eta \quad \Leftrightarrow \quad \lambda \leqslant \eta / \mu .
$$

A fuzzy relation $\alpha \in L^{A \times A}$ is reflexive if $\Delta_{A} \leqslant \alpha$ (where $\Delta_{A}$ is the crisp equality on $A$ ), symmetric if $\alpha^{-1} \leqslant \alpha$, and transitive if $\alpha \circ \alpha \leqslant \alpha$. A reflexive, symmetric and transitive fuzzy relation is called a fuzzy equivalence, whereas a reflexive and transitive fuzzy relation is called a fuzzy quasi-order.

Hereinafter, $\mathbb{N}$ denotes the set of natural numbers (without zero), $\mathbb{N}^{0}=\mathbb{N} \cup\{0\}$, and $[1, n]$ denotes the set of first $n$ natural numbers, for each $n \in \mathbb{N}$. For a given family of fuzzy relations $\mathcal{R}$, by $\mathcal{L}(\mathcal{R})$ we denote the subalgebra of $\mathcal{L}$ generated by all membership values taken by fuzzy relations from $\mathcal{R}$.

Let $n \in \mathbb{N}$ and let $A_{1}, \ldots, A_{n}$ be a collection of non-empty sets. The set $L^{A_{1} \times A_{1}} \times \cdots \times L^{A_{n} \times A_{n}}$ of all $n$-tuples of fuzzy relations on $A_{1}, \ldots, A_{n}$, respectively, is ordered pointwise, in the following way: $\left(\alpha_{1}, \ldots, \alpha_{n}\right) \leqslant\left(\beta_{1}, \ldots, \beta_{n}\right)$ if and only if $\alpha_{l} \leqslant \beta_{l}$, for each $l \in[1, n]$. Following the terminology that is used for fuzzy sets and relations, we will say that $\left(\alpha_{1}, \ldots, \alpha_{n}\right)$ is contained in $\left(\beta_{1}, \ldots, \beta_{n}\right)$.

A partially ordered set $P$ is said to satisfy the $d e$ scending chain condition, shortly $D C C$, if each descending sequence of elements of $P$ stabilizes, i.e., if $\left\{a_{k}\right\}_{k \in \mathbb{N}}$ is a sequence of elements of $P$ such that $a_{k+1} \leqslant a_{k}$, for each $k \in \mathbb{N}$, then there exists $n \in \mathbb{N}$ such that $a_{n}=a_{n+m}$, for each $m \in \mathbb{N}$.

For more information on fuzzy sets and fuzzy relations we refer to $[2,3]$.

\section{Regular fuzzy equivalences}

In this section we first define a multi-mode multirelational fuzzy network and the concept of a regular fuzzy equivalence on such a network.

Given $n \in \mathbb{N}$, a collection $A_{1}, \ldots, A_{n}$ of nonempty sets and a non-empty set $J \subseteq[1, n] \times$ $[1, n]$, and let $\left\{I_{j, k}\right\}_{(j, k) \in J}$ be also a collection of non-empty sets. We require that for each $j \in[1, n]$ there is $k \in[1, n]$ such that $(j, k) \in J$ or $(k, j) \in J$. For any $(j, k) \in J$ let $\left\{R_{i}^{j, k}\right\}_{i \in I_{j, k}}$ be a family of nonempty fuzzy relations between $A_{j}$ and $A_{k}$, and set

$$
\mathcal{R}=\left\{R_{i}^{j, k} \mid(j, k) \in J, i \in I_{j, k}\right\} .
$$

The system $\mathcal{N}=\left(A_{1}, \ldots, A_{n}, \mathcal{R}\right)$ is called a multimode multi-relational fuzzy network, or more specifically, an n-mode multi-relational fuzzy network. We usually omit the adjective "multi-relational". The sets $A_{1}, \ldots, A_{n}$ are called the components or modes of the network $\mathcal{N}$. Note that the above families of fuzzy relations are specified for some pairs of components, but not necessarily for all pairs. It is evident that $J$ is the set of all pairs $(j, k) \in[1, n] \times[1, n]$ for which a non-empty family of non-empty fuzzy relations between $A_{j}$ and $A_{k}$ is specified, and this family is indexed by the set $I_{j, k}$. If for some $j \in[1, n]$ there is no any $k \in[1, n]$ such that a non-empty family of non-empty fuzzy relations between $A_{j}$ and $A_{k}$ or between $A_{k}$ and $A_{j}$ is specified, then $A_{j}$ is isolated and there is no sense to consider it. For this reason we have introduced the above requirement for the set $J$.

Observe that the multi-mode network $\mathcal{N}$ is a complex system which consists of one-mode fuzzy networks $\mathcal{N}_{j}=\left(A_{j},\left\{R_{i}^{j, j}\right\}_{i \in I_{j, j}}\right)$, for those $j \in[1, n]$ for which $(j, j) \in J$, and two-mode fuzzy networks $\mathcal{N}_{j, k}=\left(A_{j}, A_{k},\left\{R_{i}^{j, k}\right\}_{i \in I_{j, k}}\right)$, for those $(j, k) \in J$ for which $j \neq k$. Therefore, for $n=1$ this definition gives a one-mode fuzzy network, and for $n=2$ and $J=\{(1,2)\}$ it gives a two-mode fuzzy network.

For any $l \in[1, n]$ let $\alpha_{l}$ be a fuzzy equivalence on $A_{l}$ such that for all $(j, k) \in J, i \in I_{j, k}$ the following is true:

$$
\alpha_{j} \circ R_{i}^{j, k}=R_{i}^{j, k} \circ \alpha_{k} .
$$

Then the $n$-tuple $\left(\alpha_{1}, \ldots, \alpha_{n}\right)$ is said to be a regular fuzzy equivalence on the network $\mathcal{N}$. In addition, if $\alpha_{1}, \ldots, \alpha_{n}$ are crisp equivalences, then we say that $\left(\alpha_{1}, \ldots, \alpha_{n}\right)$ is a regular crisp equivalence on $\mathcal{N}$. It is clear that $\left(\alpha_{1}, \ldots, \alpha_{n}\right)$ is a regular fuzzy equivalence on $\mathcal{N}$ if and only if $\alpha_{j}$ is a regular fuzzy equivalence on the one-mode network $\mathcal{N}_{j}$, for each $j \in[1, n]$ such that $(j, j) \in J$, and the pair $\left(\alpha_{j}, \alpha_{k}\right)$ is a regular fuzzy equivalence on the two-mode network $\mathcal{N}_{j, k}$ for each $(j, k) \in J$ for which $j \neq k$. The role of the regular fuzzy equivalence $\left(\alpha_{1}, \ldots, \alpha_{n}\right)$ can be understood as follows: for any $j \in[1, n]$ the fuzzy equivalence $\alpha_{j}$ identifies and classifies similar entities in the component $A_{j}$, entities that occupy the same position or play the same role in the network $\mathcal{N}$.

By the first theorem we prove the existence of the greatest regular fuzzy equivalence contained in a given $n$-tuple of fuzzy equivalences on the components of a multi-mode fuzzy network. We also show that it is the greatest solution, contained the same $n$-tuple of fuzzy equivalences, to a particular system of fuzzy relation equations.

Theorem 3.1 Let $\mathcal{N}=\left(A_{1}, \ldots, A_{n}, \mathcal{R}\right)$ be a multimode fuzzy network, for each $l \in[1, n]$ let $\xi_{l}$ denote an unknown taking values in $L^{A_{l} \times A_{l}}$, and let $\left(\alpha_{1}^{0}, \ldots, \alpha_{n}^{0}\right)$ be a given $n$-tuple of fuzzy equivalences on $A_{1}, \ldots, A_{n}$, respectively. Then the system of fuzzy relation equations

$$
\begin{aligned}
\xi_{j} \circ R_{i}^{j, k} & =R_{i}^{j, k} \circ \xi_{k}, \quad(j, k) \in J, i \in I_{j, k}, \\
\xi_{j}^{-1} \circ R_{i}^{j, k} & =R_{i}^{j, k} \circ \xi_{k}^{-1}, \quad(j, k) \in J, i \in I_{j, k},
\end{aligned}
$$

has the greatest solution contained in $\left(\alpha_{1}^{0}, \ldots, \alpha_{n}^{0}\right)$, which is the greatest regular fuzzy equivalence on $\mathcal{N}$ contained in $\left(\alpha_{1}^{0}, \ldots, \alpha_{n}^{0}\right)$.

Proof. It is easy to see that the system (8)-(9) has at least one solution contained in $\left(\alpha_{1}^{0}, \ldots, \alpha_{n}^{0}\right)$, the $n$-tuple $\left(\Delta_{A_{1}}, \ldots, \Delta_{A_{n}}\right)$ consiting of equality relations on $A_{1}, \ldots, A_{n}$. 
Let $\left\{\left(\alpha_{1}^{t}, \ldots, \alpha_{n}^{t}\right)\right\}_{t \in T}$ be the family of all solutions to (8)-(9) contained in $\left(\alpha_{1}^{0}, \ldots, \alpha_{n}^{0}\right)$, and for any $l \in[1, n]$ let

$$
\alpha_{l}=\bigvee_{t \in T} \alpha_{l}^{t}
$$

Then for all $(j, k) \in J$ and $i \in I_{j, k}$ we have that,

$$
\begin{aligned}
\alpha_{j} \circ R_{i}^{j, k} & =\left(\bigvee_{t \in T} \alpha_{j}^{t}\right) \circ R_{i}^{j, k}=\bigvee_{t \in T}\left(\alpha_{j}^{t} \circ R_{i}^{j, k}\right) \\
& =\bigvee_{t \in T}\left(R_{i}^{j, k} \circ \alpha_{k}^{t}\right)=R_{i}^{j, k} \circ\left(\bigvee_{t \in T} \alpha_{k}^{t}\right) \\
& =R_{i}^{j, k} \circ \alpha_{k},
\end{aligned}
$$

and similarly we prove that $\alpha_{j}^{-1} \circ R_{i}^{j, k}=R_{i}^{j, k} \circ \alpha_{k}^{-1}$, for all $(j, k) \in J$ and $i \in I_{j, k}$. Thus, $\left(\alpha_{1}, \ldots, \alpha_{n}\right)$ is a solution to the system (8)-(9), and evidently, it is the greatest solution to this system contained in $\left(\alpha_{1}^{0}, \ldots, \alpha_{n}^{0}\right)$.

Further, it is easy to verify that $\left(\Delta_{A_{1}}, \ldots, \Delta_{A_{n}}\right)$, $\left(\alpha_{1}^{-1}, \ldots, \alpha_{n}^{-1}\right)$ and $\left(\alpha_{1} \circ \alpha_{1}, \ldots, \alpha_{n} \circ \alpha_{n}\right)$ are also solutions to (8)-(9). Since $\left(\alpha_{1}, \ldots, \alpha_{n}\right)$ is the greatest solution to this system contained in $\left(\alpha_{1}^{0}, \ldots, \alpha_{n}^{0}\right)$, we conclude that $\Delta_{A_{l}} \leqslant \alpha_{l}, \alpha_{l}^{-1} \leqslant \alpha_{l}$ and $\alpha_{l} \circ \alpha_{l} \leqslant \alpha_{l}$, which means that $\alpha_{l}$ is a fuzzy equivalence, for every $l \in[1, n]$, and therefore, the $n$-tuple $\left(\alpha_{1}, \ldots, \alpha_{n}\right)$ is a regular fuzzy equivalence on the network $\mathcal{N}$.

Finally, every regular fuzzy equivalence on $\mathcal{N}$ is a solution to (8)-(9), so we conclude that $\left(\alpha_{1}, \ldots, \alpha_{n}\right)$ is the greatest regular fuzzy equivalence on $\mathcal{N}$ contained in $\left(\alpha_{1}^{0}, \ldots, \alpha_{n}^{0}\right)$.

Note that regular fuzzy equivalences are $n$-tuples of fuzzy equivalences that are solutions to system (8). However, the greatest solution to this system, contained in a given $n$-tuple of fuzzy equivalences, is an $n$-tuple of fuzzy quasi-orders, but it is not necessarily an $n$-tuple of fuzzy equivalences. To ensure that this greatest solution is an $n$-tuple of fuzzy equivalences, the system (8) must be combined with the system (9), as was done in the previous theorem.

Next, for each $j \in[1, n]$ we set

$$
\begin{aligned}
& \Lambda_{j}=\{k \in[1, n] \mid(j, k) \in J\}, \\
& P_{j}=\{k \in[1, n] \mid(k, j) \in J\} .
\end{aligned}
$$

Moreover, for a given family $\mathcal{F}$ of fuzzy relations, $\mathcal{L}(\mathcal{F})$ denotes the subalgebra of $\mathcal{L}$ generated by all membership values taken by fuzzy relations from $\mathcal{F}$.

The following theorem gives a procedure for computing the greatest regular fuzzy equivalence contained in a given tuple of fuzzy equivalences.

Theorem 3.2 Let $\mathcal{N}=\left(A_{1}, \ldots, A_{n}, \mathcal{R}\right)$ be a multimode fuzzy network, and let $\left(\alpha_{1}^{0}, \ldots, \alpha_{n}^{0}\right)$ be a given $n$-tuple of fuzzy equivalences on $A_{1}, \ldots, A_{n}$, respectively, and let $\left\{\left(\alpha_{1}^{r}, \ldots, \alpha_{n}^{r}\right)\right\}_{r \in \mathbb{N}}$ be a descending sequence of $n$-tuples of fuzzy relations on $A_{1}, \ldots, A_{n}$, respectively, defined inductively as follows:

$$
\begin{aligned}
\left(\alpha_{1}^{1}, \ldots, \alpha_{n}^{1}\right)= & \left(\alpha_{1}^{0}, \ldots, \alpha_{n}^{0}\right), \\
\alpha_{j}^{r+1}=\alpha_{j}^{r} \wedge & \bigwedge_{k \in \Lambda_{j}} \bigwedge_{i \in I_{j, k}}\left(\left[\left(R_{i}^{j, k} \circ \alpha_{k}^{r}\right) / R_{i}^{j, k}\right] \wedge\right. \\
& \left.\wedge\left[\left(R_{i}^{j, k} \circ\left(\alpha_{k}^{r}\right)^{-1}\right) / R_{i}^{j, k}\right]^{-1}\right) \wedge \\
\wedge & \bigwedge_{k \in P_{j}} \bigwedge_{i \in I_{k, j}}\left(\left[R_{i}^{k, j} \backslash\left(\alpha_{k}^{r} \circ R_{i}^{k, j}\right)\right] \wedge\right. \\
& \left.\left.\wedge\left[R_{i}^{k, j} \backslash\left(\left(\alpha_{k}^{r}\right)^{-1}\right) \circ R_{i}^{k, j}\right)\right]^{-1}\right),
\end{aligned}
$$

for all $j \in[1, n], r \in \mathbb{N}$. Then the following is true:

(a) if there exists $s \in \mathbb{N}$ such that

$$
\left(\alpha_{1}^{s}, \ldots, \alpha_{n}^{s}\right)=\left(\alpha_{1}^{s+1}, \ldots, \alpha_{n}^{s+1}\right),
$$

then the $n$-tuple $\left(\alpha_{1}^{s}, \ldots, \alpha_{n}^{s}\right)$ is the greatest regular fuzzy equivalence on the fuzzy network $\mathcal{N}$ contained in $\left(\alpha_{1}^{0}, \ldots, \alpha_{n}^{0}\right)$;

(b) if $A_{1}, \ldots, A_{n}$ are finite sets and the subalgebra $\mathcal{L}\left(\mathcal{R} \cup\left\{\alpha_{1}^{0}, \ldots, \alpha_{n}^{0}\right\}\right)$ satisfies $D C C$, then the sequence $\left\{\left(\alpha_{1}^{r}, \ldots, \alpha_{n}^{r}\right)\right\}_{r \in \mathbb{N}}$ is finite and there is $s \in \mathbb{N}$ so that $\left(\alpha_{1}^{s}, \ldots, \alpha_{n}^{s}\right)=\left(\alpha_{1}^{s+1}, \ldots, \alpha_{n}^{s+1}\right)$.

Proof. (a) Suppose that there exists $s \in \mathbb{N}$ such that $\left(\alpha_{1}^{s}, \ldots, \alpha_{n}^{s}\right)=\left(\alpha_{1}^{s+1}, \ldots, \alpha_{n}^{s+1}\right)$. Then we have that

$$
\alpha_{j}^{s} \leqslant\left(R_{i}^{j, k} \circ \alpha_{k}^{s}\right) / R_{i}^{j, k}
$$

and

$$
\left(\alpha_{j}^{s}\right)^{-1} \leqslant\left(R_{i}^{j, k} \circ\left(\alpha_{k}^{s}\right)^{-1}\right) / R_{i}^{j, k},
$$

for all $j \in[1, n], k \in \Lambda_{j}$ and $i \in I_{j, k}$, and also,

$$
\alpha_{j}^{s} \leqslant R_{i}^{k, j} \backslash\left(\alpha_{k}^{s} \circ R_{i}^{k, j}\right)
$$

and

$$
\left(\alpha_{j}^{s}\right)^{-1} \leqslant R_{i}^{k, j} \backslash\left(\left(\alpha_{k}^{s}\right)^{-1} \circ R_{i}^{k, j}\right),
$$

for all $j \in[1, n], k \in P_{j}$ and $i \in I_{k, j}$. According to the residuation property, this is equivalent to

$$
\alpha_{j}^{s} \circ R_{i}^{j, k} \leqslant R_{i}^{j, k} \circ \alpha_{k}^{s}
$$

and

$$
\left(\alpha_{j}^{s}\right)^{-1} \circ R_{i}^{j, k} \leqslant R_{i}^{j, k} \circ\left(\alpha_{k}^{s}\right)^{-1},
$$

for all $j \in[1, n], k \in \Lambda_{j}$ and $i \in I_{j, k}$, and also,

$$
R_{i}^{k, j} \circ \alpha_{j}^{s} \leqslant \alpha_{k}^{s} \circ R_{i}^{k, j}
$$

and

$$
R_{i}^{k, j} \circ\left(\alpha_{j}^{s}\right)^{-1} \leqslant\left(\alpha_{k}^{s}\right)^{-1} \circ R_{i}^{k, j},
$$

for all $j \in[1, n], k \in P_{j}$ and $i \in I_{k, j}$. It is not hard to verify that the system consisting of the previous four types of fuzzy relation inequalities is equivalent to the system (8)-(9), and consequently, the $n$-tuple $\left(\alpha_{1}^{s}, \ldots, \alpha_{n}^{s}\right)$ is a solution to the system (8)-(9). Evidently, it is contained in $\left(\alpha_{1}^{0}, \ldots, \alpha_{n}^{0}\right)$.

Let $\left(\alpha_{1}, \ldots, \alpha_{n}\right)$ be an arbitrary solution to the system (8)-(9) contained in $\left(\alpha_{1}^{0}, \ldots, \alpha_{n}^{0}\right)$. Suppose that $\left(\alpha_{1}, \ldots, \alpha_{n}\right) \leqslant\left(\alpha_{1}^{r}, \ldots, \alpha_{n}^{r}\right)$, for some $r \in \mathbb{N}^{0}$. 
Then for each $j \in[1, n], k \in \Lambda_{j}$ and $i \in I_{j, k}$ we have that

$$
\alpha_{j} \circ R_{i}^{j, k} \leqslant R_{i}^{j, k} \circ \alpha_{k} \leqslant R_{i}^{j, k} \circ \alpha_{k}^{r},
$$

whence

$$
\alpha_{j} \leqslant\left(R_{i}^{j, k} \circ \alpha_{k}^{r}\right) / R_{i}^{j, k},
$$

and similarly,

$$
\left(\alpha_{j}\right)^{-1} \leqslant\left(R_{i}^{j, k} \circ\left(\alpha_{k}^{r}\right)^{-1}\right) / R_{i}^{j, k},
$$

which implies that

$$
\begin{aligned}
\alpha_{j} \leqslant \alpha_{j}^{r} \wedge \bigwedge_{k \in \Lambda_{j}} \bigwedge_{i \in I_{j, k}}\left(\left[\left(R_{i}^{j, k} \circ \alpha_{k}^{r}\right) / R_{i}^{j, k}\right] \wedge\right. \\
\left.\wedge\left[\left(R_{i}^{j, k} \circ\left(\alpha_{k}^{r}\right)^{-1}\right) / R_{i}^{j, k}\right]^{-1}\right) .
\end{aligned}
$$

In the same way we show that

$$
\begin{aligned}
\alpha_{j} \leqslant \alpha_{j}^{r} \wedge \bigwedge_{k \in P_{j}} \bigwedge_{i \in I_{k, j}}\left(\left[R_{i}^{k, j} \backslash\left(\alpha_{k}^{r} \circ R_{i}^{k, j}\right)\right] \wedge\right. \\
\left.\left.\wedge\left[R_{i}^{k, j} \backslash\left(\left(\alpha_{k}^{r}\right)^{-1}\right) \circ R_{i}^{k, j}\right)\right]^{-1}\right) .
\end{aligned}
$$

Now, according to (11) we have that $\alpha_{j} \leqslant \alpha_{j}^{r+1}$, for each $j \in[1, n]$, and by induction we conclude that $\alpha_{j} \leqslant \alpha_{j}^{r}$, for all $j \in[1, n]$ and $r \in \mathbb{N}^{0}$.

Consequently, $\alpha_{j} \leqslant \alpha_{j}^{s}$, for each $j \in[1, n]$, which means that the $n$-tuple $\left(\alpha_{1}^{s}, \ldots, \alpha_{n}^{s}\right)$ is the greatest solution to the system (8)-(9) contained in the $n$ tuple $\left(\alpha_{1}^{0}, \ldots, \alpha_{n}^{0}\right)$. Now, according to Theorem 3.1, we conclude that $\left(\alpha_{1}^{s}, \ldots, \alpha_{n}^{s}\right)$ is the greatest regular fuzzy equivalence on $\mathcal{N}$ contained in $\left(\alpha_{1}^{0}, \ldots, \alpha_{n}^{0}\right)$.

(b) Suppose that $A_{1}, \ldots, A_{n}$ are finite sets and the subalgebra $\mathcal{L}\left(\mathcal{R} \cup\left\{\alpha_{1}^{0}, \ldots, \alpha_{n}^{0}\right\}\right)$ satisfies DCC.

For each $l \in[1, n]$ and all pairs $\left(a_{l}, a_{l}^{\prime}\right) \in A_{l} \times A_{l}$, we have that $\left\{\alpha_{l}^{r}\left(a_{l}, a_{l}^{\prime}\right)\right\}_{r \in \mathbb{N}}$ is a descending sequence in $\mathcal{L}\left(\mathcal{R} \cup\left\{\alpha_{1}^{0}, \ldots, \alpha_{n}^{0}\right\}\right)$. By the hypothesis, this sequence stabilizes, and since there are finitely many such sequences, we conclude that there exists $s \in \mathbb{N}$ such that all these sequences stabilize after $s$ steps. Therefore, the sequence $\left\{\left(\alpha_{1}^{r}, \ldots, \alpha_{n}^{r}\right)\right\}_{r \in \mathbb{N}}$ is finite and $\left(\alpha_{1}^{s}, \ldots, \alpha_{n}^{s}\right)=\left(\alpha_{1}^{s+1}, \ldots, \alpha_{n}^{s+1}\right)$ for $s \in \mathbb{N}$ whose existence has been established above.

This completes the proof of the theorem.

By Theorem 3.2, the greatest regular fuzzy equivalence contained in the given $n$-tuple $\left(\alpha_{1}^{0}, \ldots, \alpha_{n}^{0}\right)$ of fuzzy equivalences can be computed as follows. We start from this $n$-tuple and build a descending sequence of $n$-tuples of fuzzy equivalences by means of the formula (11), and simultaneously we check whether two subsequent members of the sequence are equal. The procedure terminates as soon as we find the first pair of equal consecutive members of the sequence, and in this case, the last computed $n$ tuple is the greatest regular fuzzy equivalence contained in $\left(\alpha_{1}^{0}, \ldots, \alpha_{n}^{0}\right)$.

However, in general, the above described procedure do not necessarily terminate in a finite number of steps, and Theorem 3.2 provides a sufficient condition under which it will terminate, when the subalgebra $\mathcal{L}\left(\mathcal{R} \cup\left\{\alpha_{1}^{0}, \ldots, \alpha_{n}^{0}\right\}\right)$ satisfies the descending chain condition. In particular, this condition is fulfilled if $\mathcal{L}$ is a locally finite algebra, that is, if every finitely generated subalgebra of $\mathcal{L}$ is finite. The most widely used locally finite structures are the Boolean structure and the Gödel structure. For more information on local finiteness in t-norm based structures we refer to the recent paper [13].

In cases when the above procedure does not terminate in a finite number of steps and can not be used to efficiently compute the greatest regular fuzzy equivalence on the network $\mathcal{N}$ contained in $\left(\alpha_{1}^{0}, \ldots, \alpha_{n}^{0}\right)$, it is possible to modify this procedure to compute the greatest regular crisp equivalence contained in $\left(\alpha_{1}^{0}, \ldots, \alpha_{n}^{0}\right)$. This modified procedure is provided by the following theorem.

Theorem 3.3 Let $\mathcal{N}=\left(A_{1}, \ldots, A_{n}, \mathcal{R}\right)$ be a multimode fuzzy network, and let $\left(\alpha_{1}^{0}, \ldots, \alpha_{n}^{0}\right)$ be a given $n$-tuple of fuzzy equivalences on $A_{1}, \ldots, A_{n}$, respectively, and let $\left\{\left(\varrho_{1}^{r}, \ldots, \varrho_{n}^{r}\right)\right\}_{r \in \mathbb{N}}$ be a descending sequence of $n$-tuples of crisp relations on $A_{1}, \ldots, A_{n}$, respectively, defined inductively as follows:

$$
\begin{aligned}
& \left(\varrho_{1}^{1}, \ldots, \varrho_{n}^{1}\right)=\left(\left[\alpha_{1}^{0}\right]^{c}, \ldots,\left[\alpha_{n}^{0}\right]^{c}\right), \\
& \varrho_{j}^{r+1}=\varrho_{j}^{r} \wedge\left[\Phi\left(\varrho_{j}^{r}\right)\right]^{c},
\end{aligned}
$$

where

$$
\begin{aligned}
\Phi\left(\varrho_{j}^{r}\right)=\bigwedge_{k \in \Lambda_{j}} & \bigwedge_{i \in I_{j, k}}\left(\left[\left(R_{i}^{j, k} \circ \alpha_{k}^{r}\right) / R_{i}^{j, k}\right] \wedge\right. \\
& \left.\wedge\left[\left(R_{i}^{j, k} \circ\left(\alpha_{k}^{r}\right)^{-1}\right) / R_{i}^{j, k}\right]^{-1}\right) \wedge \\
\wedge & \bigwedge_{k \in P_{j}} \bigwedge_{i \in I_{k, j}}\left(\left[R_{i}^{k, j} \backslash\left(\alpha_{k}^{r} \circ R_{i}^{k, j}\right)\right] \wedge\right. \\
& \left.\left.\wedge\left[R_{i}^{k, j} \backslash\left(\left(\alpha_{k}^{r}\right)^{-1}\right) \circ R_{i}^{k, j}\right)\right]^{-1}\right),
\end{aligned}
$$

for all $j \in[1, n], r \in \mathbb{N}$.

Then there exists $s \in \mathbb{N}$ such that

$$
\left(\varrho_{1}^{s}, \ldots, \varrho_{n}^{s}\right)=\left(\varrho_{1}^{s+1}, \ldots, \varrho_{n}^{s+1}\right),
$$

and the $n$-tuple $\left(\varrho_{1}^{s}, \ldots, \varrho_{n}^{s}\right)$ is the greatest regular crisp equivalence on the fuzzy network $\mathcal{N}$ contained in $\left(\alpha_{1}^{0}, \ldots, \alpha_{n}^{0}\right)$.

The proof of this theorem is similar to the proof of Theorem 3.2 and it will be omitted. The reader can also take a look at Proposition 5.8 [15].

\section{Concluding remarks}

In this article we dealt with regular fuzzy equivalences on multi-mode fuzzy networks because such equivalences are generally recognized in the social network analysis as a powerful mean for identifying, describing and understanding the social positions. The subject of our further research will be certain more general types of fuzzy equivalences and fuzzy quasi-orders, which will also be obtained as solutions to particular systems of fuzzy relation equations and inequalities, and which we will also try to 
apply in the study of the social positions. As noted in [5], which equivalence relation is interesting to consider depends on the problem at hand, and it is likely necessary to consider several different equivalence relations for a given network, in order to understand it completely. For example, in the mentioned paper [5] the authors applied the so-called simulation equivalences in the analysis of a network that represents the communication between a group of terrorists with different social positions, and they have shown that this kind of equivalences may help to identify some social positions that cannot be recognized using regular equivalences.

\section{Acknowledgement}

This research was supported by the Ministry of Education, Science and Technological Development, Republic of Serbia, Grant No. 174013.

\section{References}

[1] V. Batagelj, Social Network Analysis, LargeScale, in R. A. Meyers (Ed.), Encyclopedia of Complexity and Systems Science, Springer, New York, pp. 8245-8265, 2009.

[2] R. Bělohlávek, Fuzzy Relational Systems: Foundations and Principles, Kluwer, New York, 2002.

[3] R. Bělohlávek and V. Vychodil, Fuzzy Equational Logic, Studies in Fuzziness and Soft Computing, Springer, Berlin-Heidelberg, 2005.

[4] S. P. Borgatti, Social Network Analysis, TwoMode Concepts in, in R. A. Meyers (Ed.), Encyclopedia of Complexity and Systems Science, Springer, New York, pp. 8279-8291, 2009.

[5] J. Brynielsson, L. Kaati and P. Svenson, Social positions and simulation relations, Social Network Analysis and Mining, 2:39-52, Springer, 2012.

[6] M. Ćirić and S. Bogdanović, Fuzzy social network analysis, Godišnjak Učiteljskog fakulteta $u$ Vranju, 1:179-190, University of Niš, Teachers Training Faculty Vranje, 2010.

[7] P. Doreian, Positional analysis and blockmodeling, in: R. A. Meyers (Ed.), Encyclopedia of Complexity and Systems Science, Springer, New York, pp. 6913-6927, 2009.

[8] P. Doreian, V. Batagelj, A. Ferligoj, Generalized blockmodeling of twomode network data, Social Networks 26:29-53. Elsevier, 2004.

[9] P. Doreian, V. Batagelj, A. Ferligoj, Generalized Blockmodeling, Cambridge University Press, 2005.

[10] T.F. Fan and C.J. Liau, Logical characterizations of regular equivalence in weighted social networks, Artiffcial Intelligence 214:66-88, Elsevier, 2014.

[11] T.F. Fan, C.J. Liau and T.Y. Lin, Positional analysis in fuzzy social networks, in: Proceed- ings of the 3rd IEEE International Conference on Granular Computing, pp 423-428, IEEE, 2007.

[12] T.F. Fan, C.J. Liau and T.Y. Lin, A theoretical investigation of regular equivalences for fuzzy graphs, International Journal of Approximate Reasoning 49(3):678-688, Elsevier, 2008.

[13] S. Gottwald, Local and relativized local finiteness in t-norm based structures, Information Sciences 228:26-36, Elsevier, 2013.

[14] J. Ignjatović and M. Ćirić, Weakly linear systems of fuzzy relation inequalities and their applications: A brief survey, Filomat, 26(2):207241, University of Niš, Faculty of Science and Mathematics, 2012.

[15] J. Ignjatović, M. Ćirić and S. Bogdanović, On the greatest solutions to weakly linear systems of fuzzy relation inequalities and equations, Fuzzy Sets and Systems 161:3081-3113, Elsevier, 2010.

[16] J. Ignjatović, M. Ćirić, N. Damljanović and I. Jančić, Weakly linear systems of fuzzy relation inequalities: The heterogeneous case, Fuzzy Sets and Systems, 199:64-91, Elsevier, 2012.

[17] F. Lorrain and H. C. White, Structural equivalence of individuals in social networks, Journal of Mathematical Sociology, 1:49-80, Taylor \& Francis, 1971.

[18] J. Scott, Social Network Analysis, Overview of, in: R. A. Meyers (Ed.), Encyclopedia of Complexity and Systems Science, Springer, New York, pp. 8265-8279, 2009.

[19] I. Stanković, M. Ćirić and J. Ignjatović, Fuzzy relation inequalities and equations with two unknowns and their applications, to appear.

[20] I. Stanković, J. Ignjatović and M. Ćirić, Boolean relation equations in data analysis, in: Proceedings of the 9th IEEE International Symposium on Intelligent Systems and Informatics (SISY 2011), Subotica, Serbia, pp. 125130, IEEE, 2011.

[21] D. R. White and K. P. Reitz, Graph and semigroup homomorphisms on networks and relations, Social Networks, 5:143-234, Elsevier, 1983.

[22] A. Žiberna, Blockmodeling of multilevel networks, Social Networks, 39:46-61, Elsevier, 2014 . 